\title{
Improved Position Estimation in Wireless Heterogeneous Networks
}

\author{
Erwan Ermel ${ }^{1,2}$, Anne Fladenmuller ${ }^{1}$, Guy Pujolle ${ }^{1}$, and André Cotton ${ }^{2}$ \\ 1 Université Pierre et Marie Curie, Laboratoire d'Informatique de Paris 6 (LIP6), \\ 8 rue du Capitaine Scott, 75005 Paris, France \\ tel:+33(0)144278879 - fax: +33(0)144277495 \\ \{Erwan.Ermel, Anne.Fladenmuller, Guy.Pujolle\}@lip6.fr \\ 2 Thales Communication, BGCOM/TCF/SAS/SEA/TAI, \\ 160 bld de Valmy - BP 82, 92704 Colombes Cedex, France \\ tel: $+33(0) 146132216$ - fax: $+33(0) 146132668$ \\ Andre.Cotton@fr. thalesgroup.com
}

\begin{abstract}
This paper addresses the problem of nodes localization in wireless ad hoc networks. Two types of nodes are considered: nodes with self-locating capability like GPS and nodes with no self-locating capability. For the last ones it is thus important to infer a position which will be retrieved from the position of the neighbor's nodes. The precision of this information clearly depends on the environment and may not be very accurate. We propose a method which consists in selecting and processing only nodes that are likely to enhance the accuracy of an estimated position. We focus our approach on defining a hull, made up of neighboring nodes, as a key element of position accuracy enhancement. The improvements of using such a method are then validated by a set of simulations.
\end{abstract}

\section{Introduction}

Ad hoc networks consist of wireless heterogeneous nodes that communicate with each other without any pre-established infrastructure. These autonomous networks focus on providing self-configuring networks that are easily and quickly deployable. Ad hoc networks are of great interest and have a wide range of applications, as for in war theatres or disasters relief.

Due to the wide diversity of wireless devices available on the market and the various capabilities they offer, it seems restrictive to suppose that all nodes will possess a localization system such as GPS [1] or Galileo [2]. Thus, nodes without such type of equipment will have to retrieve a geographical position. Such information can useful for many purposes: geographical routing, location services or more generally in ambient networks.

The localization process can be fully distributed, based on the information a node can get from its neighbors position, whether this position has itself been inferred by a localization process or obtained thanks to dedicated devices. The purpose of this paper is thus to propose a method to improve the precision 
of the position estimation in the most generic environments such as a network composed of basic laptops with simple WiFi cards.

Most position estimation techniques are based on geometrical computations like triangulation or trilateration. To evaluate the distance between two nodes, several methods can be used. Four classes of position estimation methods can be defined: the first one consists in determining the time-of-flight of a signal between two anchors (Time Of Arrival [3,4] and Time Difference Of Arrival [5,6]). The second class is based on the signal strength [7,8]: when the emission power is known, the distance to the anchor can be approximated. The third class is based on triangulation like the Angle of Arrival (AoA) estimates the direction of an incoming signal from several anchors, and then estimates the position. As for the fourth, it gathers all remaining position estimation methods like connectivity based approach $[9,10,11]$. Our approach belongs to the fourth and last class.

We define two classes of nodes: self-locating nodes, which are embedded with self-locating capability, such as, and simple nodes with no self-locating capability. We call anchor or landmark a node that knows its position. In this paper, we investigate a simple method to select nodes with the aim to enhance the accuracy of estimated positions. Our technique can be implemented and used by every position estimation methods stated above as it is a step just before the real position estimation process.

The structure of this paper is done as follow. We first present the assumptions and the definition made in this paper. Section 3 details the hull method to select anchors within neighboring nodes, followed in Section 4 by our simulation results. Section 5 concludes the paper.

\section{Assumptions and Definitions}

We limit our approach to select only one-hop anchors but this technique is also feasible for $n$-hops nodes selection. No distance measurement is to be used to estimate the position of a simple node. Thus a node only exploits its neighbor's nodes connectivity.

Let $S$ be a simple node. Let $S_{\text {est }}$ be the estimated position of the node $S$, and $S_{\text {real }}$ be the coordinates of its real location. Note that $S_{\text {real }}$ information is only used by simulations to evaluate the precision of our algorithm. We can not possess such information in reality as it represents what we are looking for. Let $R_{\max }$ be the maximum theoretical transmission range of $S$. We also define the accuracy of the node position $C_{a c c}$, as a function of the localization error represented by the distance between $S_{\text {real }}$ and $S_{\text {est }}$ :

$$
C_{a c c}=1-\frac{\left\|\left(S_{r e a l}, S_{e s t}\right)\right\|_{2}}{R_{\max }}
$$

By definition, $0 \leq C_{a c c} \leq 1$. Self-locating nodes with accurate coordinates, like the ones given by a GPS have a position accuracy of 1 . On the other side, simple nodes, which have to estimate their position, have a position accuracy $0 \leq C_{a c c}<1$. Note that these simple nodes don't only have to retrieve their 
position coordinates but also to evaluate the precision of this information. In order to do so, several methods such as statistical or area computing approaches are detailed in [12].

\section{Convex Hull Selection}

Our main goal in this paper is to enhance the accuracy of an estimated position by selecting only anchors that are likely to improve the position estimation process. We detail in this section a simple approach to select anchors amount the one-hop nodes in a wireless networks: the convex hull selection.

Computational geometry deals with geometrical problems. These problems are for example convex hull among a list of nodes, Voronoï diagrams, geometric searching. These algorithms are well detailed in $[13,14,15,16]$.

The main idea of using a convex hull as a selection method among nodes is to choose only nodes which are at the greatest distance from anchors. As the position estimation process is based on trilateration, the further apart the anchors are, the better will be the accuracy of the estimated position.

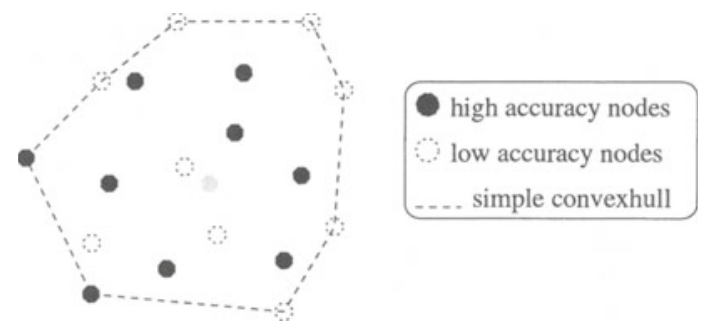

Fig. 1. Convex hull: simple convex hull considers the distance metric to elect hull nodes.

We choose the convex hull approach as our nodes selection method. Only the hull nodes are taken into account in the position estimation process. The remaining nodes are simply discarded. A convex hull example is shown in Fig. 1.

The convex hull of a set of points $S$ in n dimensions is the intersection of all convex sets containing $S$. For $N$ nodes $n_{1}, \ldots, n_{N}$, the convex hull $C$ is then given by the expression:

$$
C \equiv\left\{\sum_{j=1}^{N} \lambda_{j} n_{j}: \lambda_{j} \geq 0 \text { for all } j \text { and } \sum_{j=1}^{N} \lambda_{j}=1\right\} .
$$

The simple convex hull selects only the nodes for their physical position whatever their position accuracy $C_{a c c}$ is. We will study in Section 4 the performances of this simple hull selection method. 


\section{Evaluation of the Hull Selection}

The simulations were performed under Java. 50 nodes were randomly placed in a $1000 \mathrm{~m} \times 1000 \mathrm{~m}$ square. Self-locating nodes and simple nodes were also randomly elected. The maximum theoretical transmission range $R_{\max }$ was set to $170 \mathrm{~m}$.

Fig.2 shows the impact of our simple hull selection method on the accuracy of an estimated position. To compare our selection method, we choose as the reference model the greedy scheme: all the neighbors nodes are selected in the position estimation process. None are discarded. The estimated position is obtained by a simple centroid formula, where all the nodes have got the same weight.

We want here to compare the impact of choosing carefully some nodes from our neighborhood (simple hull method) or of getting as much information as we can (greedy method) to enter the position estimation process. The simulations provide us the accuracy of the estimated position by monitoring the distance between the real and the estimated positions. For each selection method (hull or greedy), we keep track of the number of neighbors and their type: self-locating and simple nodes. It seems important to differentiate both types as self-locating nodes have accurate position information, whereas simple nodes already have an inferred position with a much less accurate value. We thus want to evaluate the impact on the accuracy of the position to estimate another position. By running a large number of simulations, we then obtain an average accuracy for each combination of neighbors (number of simple and self-locating nodes).

In Fig. 2, the graphs plots in (a),(c),(e) (respectively (b),(d),(f)) the average accuracy of the estimated position as a function of the number of simple nodes (resp. self-locating nodes) in the neighborhood. The number of selflocating nodes (respectively simples nodes) in the direct neighborhood is set to 0 (resp. 2 and 6) for (a),(b) (resp. (c),(d) and (e),(f)).

It is obvious that the more precise the position information retrieved from the neighborhood is, the better will be the accuracy of the estimated position. Nevertheless it also appears clearly from these plots that the hull method, which consists of selecting the position information from the nodes the further apart from each other, gives better results. As for in Fig. 2 (a), where we can see for instance that selecting 3 simple nodes gives on average similar performances than taking the all 6 simple nodes in the greedy approach.

We also notice that the more sln nodes are used bye the estimation process, the better is the position accuracy.

In every case, the simple selection gives a better position accuracy than the greedy approach does. The selection enhances the position accuracy up to $20 \%$.

\section{Conclusion}

We present and compare in this paper a simple method to select anchors in a wireless network to enhance the position estimation of simple nodes, nodes with no self-locating capabilities. 


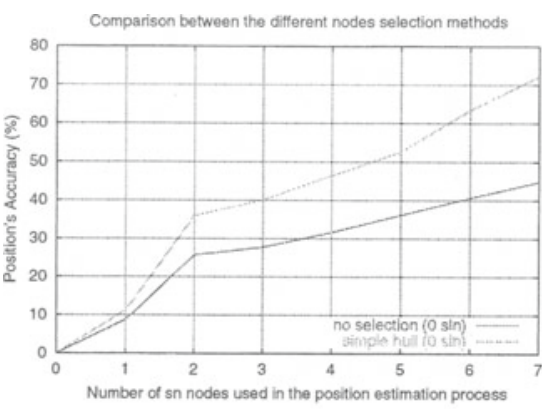

(a)

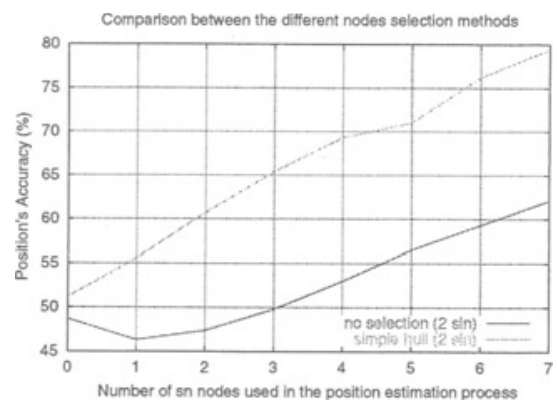

(c)

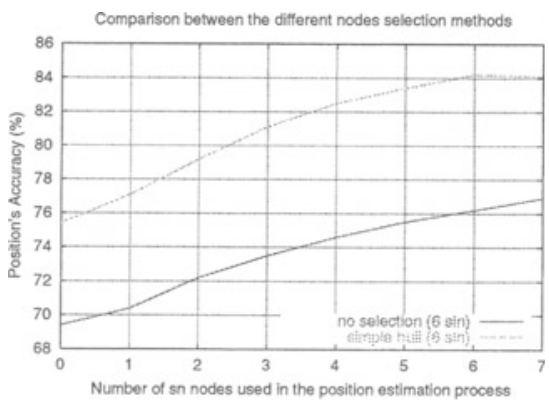

(e)

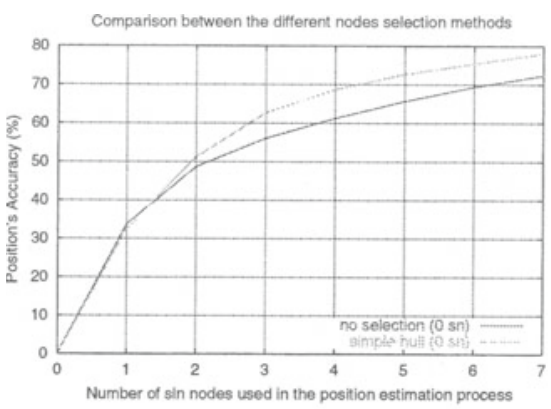

(b)

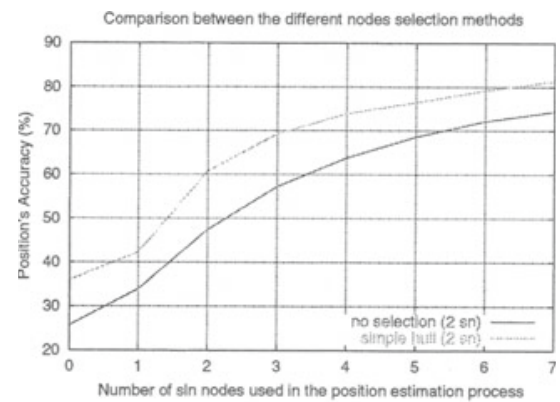

(d)

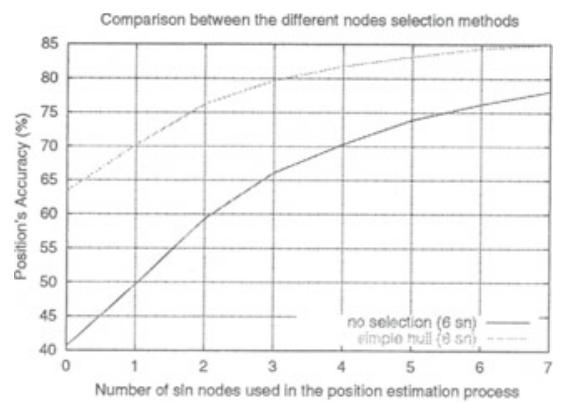

(f)

Fig. 2. Impact of nodes selection method on the average accuracy of the estimated position. 
Our nodes selection approach deals with the definition of a convex hull among neighbor nodes. The resulting position accuracy using such nodes selection method is enhancing from a greedy scheme up to $20 \%$. We also show that the accuracy of an estimated position only take advantage of the distance between the hull nodes, whatever their position accuracy is.

Our next step consists of implementing such selection algorithm in a global geographical routing protocol in a heterogeneous network under NS-2.

\section{References}

1. Hofmann-Wellenhof, B., Lichtenegger, H., Collins, J.: Global Positioning System : Theory and Practice. Springer-Verlag (1997)

2. (Galileo) http://europa.eu.int/comm/dgs/energy_transport/galileo/.

3. Capkun, S., Hamdi, M., Hubaux, J.P.: Gps-free positioning in mobile ad-hoc networks. Cluster Computing 5 (2002)

4. Werb, J., Lanzl, C.: A positioning system for finding things indoors. IEEE Spectrum 35 (1998) 71-78

5. Savvides, A., Han, C.C., Strivastava, M.B.: Dynamic fine-grained localization in ad-hoc networks of sensors. (2001)

6. Ward, A., Jones, A., Hopper, A.: A new location technique for the active office. IEEE Personal Communications 4 (1997) 42-47

7. Savarese, C., Rabaey, J.M., Beutel, J.: Localization in distributed ad-hoc wireless sensor networks. Proceedings of the ICASSP (2001)

8. Beutel, J.: Geolocalisation in a picoradio environment. Master's thesis, ETH Zurich, Electronics Lab (1999)

9. Doherty, L., Pister, K.S.J., Ghaoui, L.E.: Convex optimization methods for sensor node position estimation. In: Proceedings of IEEE INFOCOM'2001, Anchorage (2001)

10. Niculescu, D., Nath, B.: Dv based positioning in ad hoc networks. Telecommunication Systems 22 (2003) 267-280

11. Bulusu, N., Heidemann, J., Estrin, D.: Gps-less low cost outdoor localization for very small devices. IEEE Personal Communication, Special Issue on Smart Spaces and Environment 7 (2000) 28-34

12. Ermel, E., Fladenmuller, A., Pujolle, G., Cotton, A.: Estimation de positions dans des réseaux sans-fil hybrides. In: CFIP 2003. (2003)

13. Preparata, F.P., Shamos, M.I.: Computational Geometry: An Introduction. Springer Verlag (1991)

14. de Berg, M., Kreveld, M., Overmars, M., Scharzkopf, O.: Computational Geometry, Algoritms and Application. Springer (1997)

15. O'Rourke, J.: Computational Geometry in C. Cambridge University Press (1998)

16. Lemaire, C.: Triangulatation de Delaunay et arbres multidimensionnels. $\mathrm{PhD}$ thesis, Ecole des mines de Saint-Etienne (1997) 